\title{
Evolution in Fine-Grained Environments I. Environmental Runs and the Evolution of Homeostasis*
}

\author{
Alan R. Templeton \\ Department of Biology, Washington University, St.Louis, Missouri 63130
}

AND

EDWARD D. RothMAN

Department of Statistics, University of Michigan, Ann Arbor, Michigan 48109

Received July 2, 1977

\begin{abstract}
In this paper we discuss the problem of evolution when individual organisms are subjected to heterogeneous environments within their own lifetimes. We first develop a model of environmental heterogeneity in which there are two discrete environmental states. Transitions between states are governed by a stochastic matrix. Next, we examine how an organism responds to this hetcrogeneity. We assume that $L$ consecutive time units of the environmental process are sampled during the normal life span of the organism, and that the individual's fitness is determined in part by a component unrelated to this heterogeneity and by other components that describe the fitness response to the heterogeneity. The fitness responses are functions of the environmental state and of how long the organism has been previously exposed to that state; i.e., fitness response is dependent upon the environmental context. We then discuss how this individually experienced heterogeneity is translated to the populational level. Finally, genetic constraints are overlaid so that the tools of population genetics may be used to make evolutionary predictions.
\end{abstract}

\section{INTRODUCTION}

The world in which we live is highly heterogeneous in both space and time. Organisms must live and reproduce in the face of possible changes in both their physical environment (e.g., weather) and their biotic environment (e.g., interactions with other organisms). Much of this immense array of environmental diversity is not controllable or even predictable by the individual organism.

* Supported by National Science Foundation Grant DEB76-16985 and ERDA Contract $\mathrm{E}(11-1)-2828$. 
In such cases, the exact array of environmental states an organism experiences throughout its lifetime must be treated as a random variable.

Another level of environmental heterogeneity emerges when dealing with a population of organisms rather than just an individual. A population may be dispersed over a spatially heterogeneous terrain such that any particular individual lives entirely in one environmental patch. Also, the environment may be identical for all individuals of a given generation, but fluctuate between generations. In either case, the environment appears to be invariable to the individual, but environmental heterogeneity is manifested at the level of the population existing in space and through time. For the purposes of this series of papers, wc will refer to the environmental heterogeneity that is manifested only at the populational level as "coarse-grained" while the environmental heterogeneity experienced by individuals within their own lifetime will be called "finegrained." Further discussion of these definitions and their relationship to other uses of the word "grain" can be found in Templeton and Rothman (1978).

Population geneticists have been very concerned with the evolutionary implications of environmental heterogeneity, as shown by the recent review articles by Hedrick et al. (1976) and Felsenstein (1976). However, most of the papers reviewed in these articles deal with "coarse-grained" heterogeneity, and the problem of the evolutionary impact of fine-grained heterogeneity has been studied much less. In this paper, we will be concerned with fine-grained environments. This necessitates a rather different modeling approach to environmental heterogeneity than that encountered in coarse-grained models in which fitness is a function of the genotype and the realized environmental state in which the genotype is expressed. In fine-grained models, a single individual is exposed to several environments, and this exposure always occurs as a temporal sequence at the individual level (even if the physical source of the variability is spatial). Yet, this individual can only have one realized fitness value. Hence, the fitness impact of an environmental state must be a function not only of the genotype and that state, but also of the past environments to which the individual has been exposed and their fitness impacts. Thus, the environmental context of the state must be part of the fitness model, which represents a major difference from coarse-grained models.

Another major difference between fine-and coarse-grained models is in the manner in which fitness heterogeneity is treated at the populational level. In most coarse-grained models, there is no problem in this regard since the fitness heterogeneity by assumption exists at the populational level. However, for fine-grained models we must be concerned with how fitness heterogeneity at the individual level is translated to the populational level since populations evolve, not individuals. In this regard, fine-grained heterogeneity has often been modeled as being "averaged out" at the populational level, causing finegrained models to be treated simply as a type of constant fitness model (e.g., Strobeck, 1975). The reasoning behind this is as follows. First, because of the 
environmental heterogeneity, individual fitness may be treated as a random variable with, say, mean $w_{i}$ and variance $\sigma_{i}{ }^{2}$ for genotype $i$. If $N$ is the population size, $P_{i}$ the frequency of genotype $i$, and all individuals independently sample the environment, then the variance of the average fitness for all individuals which share genotype $i$ (i.e., the genotype fitness that enters into the allele frequency change equation) is $\sigma_{i}{ }^{2} /\left(N P_{i}\right)$. Finally, in models that assume $N \rightarrow \infty$ (as ours will in this paper), this variance goes to zero. Thus, with independent sampling and large population size, the fitness process that governs the evolution of the population can be treated as constant despite heterogeneity at the individual level.

However, there are many important instances in which the fine-grained heterogeneity is not averaged out. First, this will not occur if the total population size $N$ is small. Second, fitness heterogeneity still exists even for an effectively infinite-sized population for genotypes that depend upon rare or newly arisen alleles such that $P_{i}$ is of the order of $1 / N$. This is an important exception since the fate of new mutants is predominately determined during the first few generations in which they are rare. The final exception occurs when the assumption of independent sampling is invalid. For example, suppose we are modeling a geographically restricted plant population such that individual plants experience fluctuations in weather conditions throughout their lifetime. However, because the population is geographically restricted, all individuals may experience essentially an identical sequence of weather states. In this case, the environmental heterogeneity at the individual level is perfectly translated to the populational level, and the variance of the genotype fitness equals the variance of the individual fitness. Consider now an intermediate case in which any two individuals of the same genotype drawn from the same generation have a correlation of $\rho$ between their fitness $(0 \leqslant \rho \leqslant 1$, since this correlation is assumed to be true for all pairs sharing the same genotype). The variance of the genotype fitness is now $\sigma_{i}{ }^{2}\left(1+\left(N P_{i}-1\right) \rho\right) /\left(N P_{i}\right)$ which goes to $\sigma_{i}{ }^{2} \rho$ as $N P_{i}$ goes to infinity. Therefore, with correlated sampling, the variance does not necessarily vanish and genotype fitness must once again be treated as a random variable. In this paper, we will concern ourselves only with infinite population models in which sampling is either independent (i.e., constant genotype fitness) or completely dependent $(\rho=1$, i.e., random genotype fitness). The other two case of finite population size and of rare alleles have already been treated in a previous paper (Templeton, 1977).

\section{ENVIRONMENTAL MODEL}

We begin with a stochastic model of environmental heterogeneity. The model we use in this paper has two discrete environmental states, say 0 and 1. During any particular time unit, the environment is either in one state or the other. 
Transitions between time units are governed by the stochastic matrix

$$
\begin{aligned}
& 0 \\
& 1
\end{aligned}\left(\begin{array}{cc}
1-\alpha & 1 \\
\beta & 1-\beta
\end{array}\right)
$$

where $1-\alpha$ is the probability of being in state 0 given that the process was in state 0 during the previous time unit, $\alpha$ is the probability of being in state 1 given 0 previously, $\beta$ is the probability of being in state 0 given 1 previously, and $1-\beta$ is the probability of being in state 1 given 1 previously. The environmental process is thus assumed to be Markovian as governed by the above transition matrix. Such a model has been used to describe rainfall successfully (Gabriel and Neumann, 1962), but it need not be limited simply to describing temporal heterogeneity in weather. The average frequencies of environments 0 and 1 over a large period of time are $p_{0}=\beta /(\alpha+\beta)$ and $p_{1}=\alpha /(\alpha+\beta)$, respectively. By choosing $\alpha$ and $\beta$ appropriately, we can describe environments differing in the average frequency of their states. Moreover, an infinite number of $\alpha$ 's and $\beta$ 's can yield identical $p_{0}$ 's and $p_{1}$ 's. Thus, we can contrast environments with the same average state frequencies, but which differ in how transitions are made between states; i.e., we can have runny environments with long sequences or runs of one state or the other very probable, or nonrunny environments where rapid alternation of states is likely. By a run, we are referring to one state making a transition to another, staying in this new state for a specified period of time, and then making the transition back to the original state. For example a run of l's of length $n$ would occur when, starting with a 0 , a transition to 1 occurs, followed by $n-1$ more 1's, and then a transition back to 0 . Note that in describing a run we not only specify an environmental state, but we also place that state in an environmental context. Starting with an initial 1 , the probability of a run of 1's of length $n$ is, from Eq. $(2.1),(1-\beta)^{n-1} \beta$. The expected duration of a run of 1's is thus $1 / \beta$. Similarly, the average run length for state 0 is $1 / \alpha$. The ability to describe runs is an important feature of this model since much of the environmental heterogeneity that is known to have great biological impact is defined in terms of runs. For example, droughts are known to affect many plant and animal species greatly; yet a drought occurs only when a run of dry days is experienced. Simply specifying the average frequency of dry days does not in general define the chances of encountering a drought. Hence, some measure of the runniness of the environment is often a critical parameter for biological problems.

\section{Environmental Response Function}

We now discuss how the individual organism experiences and responds to this environmental heterogeneity. Suppose the organism of interest samples 
a set of $L$ consecutive time units from a stationary process governed by (2.1), where $L$ represents the amount of the life span that is subjected to the environmental process being modeled. We futher assume that the environments which each generation encounters are governed by identical probabilistic rules; that is, the parameters of (2.1) are constant over time. We next assume that each individual has some inherent fitness component $C$ that is solely a function of its genotype and is unaffected by the sequence of environmental states encountered. A second component of fitness that is multiplied by $C$ to yield total fitness is affected by the environmental heterogeneity. This second component can be further subdivided into multiplicative components that depend both upon the environmental state as well as the run length of the state. This allows us the option of making the fitness impact of a particular state context dependent. Thus, a state 1 preceded by a 0 may have a very different impact than a state 1 preceded by 40 other 1's (e.g., the difference between a dry day breaking a wet spell versus a dry day extending a drought). Let these components be symbolized by $\xi_{r}$ and $\omega_{n}$, where $\xi_{r}$ is the fitness response to a run of 0 's of length $r$ and $\omega_{n}$ is the fitness response to a run of 1's of length $n$. Thus, the total fitness of the individual given a realized sequence of 0 's and l's is

$$
W=C \cdot \prod_{i} \xi_{n_{i}} \cdot \prod_{j} \omega_{r_{j}},
$$

where $i$ indexes all runs of 1 's and $j$ all runs of 0 's.

We must now couple (3.1) with (2.1) in order to treat individual fitness as a random variable. Since fitness is a function both of environmental state and of run length, we first need the probabilities for the runs of various lengths in each state out of a total of $L$ units. In this regard, we can make use of the generalized probability generating function (p.g.f.) for the number of runs of 0 's and 1's of a specified length that has been derived by Good (1973) for binary Markov processes. For the Markov process described by (2.1), Good's p.g.f. reduces to the following expression after some algebraic manipulations:

$$
\frac{\alpha K+\beta \Lambda+2 \alpha \beta K \Lambda}{(\alpha+\beta)(1-\alpha \beta K \Lambda)}
$$

where

$$
\begin{aligned}
& K=\sum_{i=1}^{\infty}(1-\beta)^{i-1} x_{i}, \\
& \Lambda=\sum_{j=1}^{\infty}(1-\alpha)^{j-1} y_{j} ;
\end{aligned}
$$

$x_{i}=$ the dummy variable that indexes runs of 1's of length $i$,

$y_{j}=$ the dummy variable that indexes runs of 0 's of length $j$. 
Equation (3.2) is the p.g.f. for any total length of one or more, so to impose the restriction that the length equals $L$, set $x_{i}=x_{i} z^{i}$ and $y_{j}=y_{j} z^{j}$ in (3.2) and extract the coefficient of $z^{L}$ from the resulting expression.

Now that we have the p.g.f., we can obtain expressions for the moments of the individual fitness. To obtain the mean of (3.1), we simply set $x_{i}=\omega_{i} z^{i}$ and $y_{j}=\xi_{j} z^{j}$ in (3.2), extract the coefficient of $z^{L}$, and multiply by $C$. We can similarly obtain the second moment about the origin by letting $x_{i}=\omega_{i}{ }^{2} z^{i}$ and $y_{j}=\xi_{j}{ }^{2} z^{j}$, extracting the coefficient of $z^{L}$, and multiplying by $C^{2}$. The variance can then be computed by subtracting the square of the mean from the second moment about the origin.

Extracting the coefficient of $z^{L}$ from (3.2) can be rather cumbersome, so an approximation may be used when $L$ is large. As shown by Feller (1957), the coefficient of $z^{L}$ in a p.g.f. for large $L$ has the form

$$
\Phi z_{1}^{-(L+1)}
$$

where, in our case, $z_{1}$ is the smallest root in absolute value of

$$
1-\alpha \beta K \Lambda=0
$$

and

$$
\Phi=(\alpha K+\beta \Lambda+2) \div\left\{(\alpha+\beta) \cdot \alpha \beta\left(\frac{\partial K}{\partial z} \Lambda+K \frac{\partial \Lambda}{\partial z}\right)\right\}
$$

evaluated at $z=z_{1}$. Given numerical values for the parameters, it is much easier to find the roots of (3.5) than to try to extract the coefficient of $z^{L}$ from (3.2) directly. Hence, the mean fitness is, setting $x_{i}=\omega_{i} z^{i}$ and $y_{j}=\xi_{j} z^{j}$ in (3.3),

$$
E(W) \simeq C \Phi\left(z_{1}\right) z_{1}^{-(L+1)} .
$$

Besides the mean and variance of the fitness, it is often necessary to have the mean of the log fitness, as we shall see in subsequent sections. One can simply convert (3.2) into a generating function for the log fitness by using the dummy variables $x_{i}=z^{i} x^{1 n \omega_{j}}$ and $y_{j}=z^{j} y^{1 n \xi_{j}}$. However, we can also solve this problem directly. Let

$$
\begin{aligned}
\delta_{n j} & =1 & & \text { if a run of l's of length } n \text { occurs at event } j \\
& =0 & & \text { otherwise. }
\end{aligned}
$$

Then,

$$
\begin{aligned}
\operatorname{Prob}\left(\delta_{n j}=1\right) & =p_{1}(1-\beta)^{L-1}, & & n=L, \\
& =p_{1}(1-\beta)^{n-1} \beta, & & j=n<L, \\
& =p_{0} \alpha \beta(1-\beta)^{n-i}, & & j=n+1, \ldots, L-1 \& n<L, \\
& =p_{0} \alpha(1-\beta)^{n-1}, & & j=L \& n<L .
\end{aligned}
$$


From (3.8), the expected number of runs of 1's of length $n$ is

$$
\begin{array}{ll}
p_{1}(1-\beta)^{L-1}=\alpha(1-\beta)^{L} 1 /(\alpha+\beta), & n=L, \\
\alpha \beta(1-\beta)^{n-1}\{2+\beta(L-n-1)\} /(\alpha+\beta), & n=1,2, \ldots, L-1 .
\end{array}
$$

A similar expression may be obtained for runs of 0 's. The log fitness is

$$
\begin{aligned}
\ln W= & \ln C+\sum_{n}\left(\ln \omega_{n}\right)(\text { no. of } 1 \text { runs of length } n) \\
& +\sum_{r}\left(\ln \xi_{r}\right)(\text { no. of } 0 \text { runs of length } r) .
\end{aligned}
$$

Hence, from (3.9), the expected log fitness is

$$
\begin{aligned}
E(\ln W)= & \ln C+\frac{L \alpha \beta}{\alpha+\beta}(\overline{\ln \omega}+\overline{\ln \xi}) \\
& +\frac{\alpha \beta}{\alpha+\beta}\left((1-\beta)^{L+1} \ln \omega_{L} / \beta+(1-\alpha)^{L+1} \ln \xi_{L} / \alpha\right) \\
& +\frac{\alpha \beta}{\alpha+\beta} \sum_{n=1}^{L}\left((2-(n+1) \beta)(1-\beta)^{n-1} \ln \omega_{n}\right. \\
& \left.+(2-(n+1) \alpha)(1-\alpha)^{n-1} \ln \xi_{n}\right),
\end{aligned}
$$

where

$$
\begin{aligned}
& \overline{\ln \omega}=\beta \sum_{n=1}^{L}(1-\beta)^{n-1} \ln \omega_{n}, \\
& \overline{\ln \xi}=\alpha \sum_{n=1}^{L}(1-\alpha)^{n-1} \ln \xi_{n} .
\end{aligned}
$$

Only the second term in (3.11) is multiplied by $L$, but the last two terms of (3.11) may be appreciable even when $L$ is large if either $\beta \rightarrow 0$ or $\alpha \rightarrow 0$; that is, when the environment is extremely runny. If the environment is not very runny and $L$ is large with respect to $\alpha \beta /(\alpha+\beta)$, we can approximate average log fitness by

$$
E(\ln W) \simeq \ln C+\frac{L \alpha \beta}{\alpha+\beta}(\overline{\ln \omega}+\overline{\ln \xi}) .
$$

The term $\overline{\ln \omega}$ is simply the average $\log$ fitness effect of a run of 1 's, and $\overline{\ln \xi}$ is the average $\log$ fitness effect of a run of 0 's. Thus, $\overline{\ln \omega}+\overline{\ln \xi}$ is the average $\log$ fitness effect when exposed to one run of 1's and one run of 0 's. The meaning of the coefficient $L \alpha \beta /(\alpha+\beta)$ is made clear when it is reexpressed as $L /(1 / \alpha+1 / \beta)$. Recall that $1 / \alpha$ is the average run length of runs of 0 's while $1 / \beta$ 
is the average run length of 1's. Hence, $1 / \alpha+1 / \beta$ is the average environmental cycle length; i.e., the average length of time it takes to go from 0 's to 1's and then return to 0's or vice versa (e.g., compare the length of the "weather cycle" described in Gabriel and Neumann (1962)). Therefore, an organism will experience approximately $L /(1 / \alpha+1 / \beta)$ such cycles during the period $L$, each with an average fitness impact of $(\overline{\ln \omega}+\overline{\ln \xi})$.

In order to examine the evolution of responses to environmental heterogeneity, we will make further assumptions about the form of the $\omega$ 's and $\xi$ 's. It has been found empirically again and again that most plants and animals can withstand short exposures of even very harsh environmental states. This occurs because most organisms have a variety of physiological and behavioral homeostatic or enantiostatic mechanisms (Mangum and Towle, 1977) that can buffer them from even extreme environments for at least a short period of time. However, difficulties emerge when prolonged exposure to a particular state occurs, even for states that are actually beneficial if they occur as short runs. For example, let 0 's correspond to wet days and 1's to dry days. Most plants need a certain exposure to both wet and dry days in order to survive and grow. Hence, each state is necessary for the plant. However, a long run of wet days could result in flooding conditions, while a long run of dry days could create a drought. Either of these consequences of long environmental runs could easily kill the plant. Thus, in a great many biological situations, it is reasonable to assume that the $\omega_{n}$ 's and $\xi_{n}$ 's are close to 1 or even above 1 for small $n$ 's (i.e., short runs of either state are selectively neutral or even beneficial), but that as $n$ increases the $\omega_{n}$ 's and $\xi_{n}$ 's begin to become less than 1 (long runs are deleterious). We can gain more insight into the biologically reasonable forms for these fitness functions by examining in greater detail the mechanisms of drought resistance in plants. A plant initially copes with dry conditions through a variety of "droughtavoidance" mechanisms such as decreasing water potential to improve water uptake, closing the stomata, reducing the transpiring surface area, etc. (Larcher, 1975). If the environmental stress lasts only a short period of time, say until time $d$, the plant experiences no measurable decline in fitness. If the exposure is more prolonged, fitness begins to decline because of the fact that the avoidance mechanisms interfere with other basic physiological functions (e.g., photosynthesis) and/or that the avoidance mechanisms themselves begin to fail so that further drought resistance depends upon the capacity of the protoplasm to endure dessication ("drought tolerance" (Larcher, 1975)). Similar avoidance and tolerance mechanisms have been described for other types of environmental stress (Larcher, 1975). For the purposes of this paper, we will define short-term homeostasis as homeostasis due to avoidance mechanisms before any fitness decline occurs (i.e., before the environmental stress lasts $d$ or more time units), and long-term homeostasis as homeostasis due to mechanisms that protect the organism from the adverse side effects of prolonged use of the avoidance mechanisms and/or due to their tolerance once the avoidance mechanisms have failed. 
For analytical purposes, we will sometimes use the following simple fitness model that captures the essence of the fitness considerations given above:

$$
\begin{aligned}
\xi_{r} & =1, & & r \leqslant d_{0}, \\
& =e^{-\lambda_{0}\left(r-d_{0}\right)}, & & r>d_{0} ; \\
\omega_{n} & =1, & & n \leqslant d_{1}, \\
& =e^{-\lambda_{1}\left(n-d_{1}\right)}, & & n>d_{1} .
\end{aligned}
$$

In this model, $d_{0}$ and $d_{1}$ measure the organism's ability to cope with the initial exposures to 0's and 1's, respectively, through short-term physiological and behavioral homeostatic or enantiostatic mechanisms. The $\lambda$ 's measure the organism's long-term homeostatic or enantiostatic abilities when subjected to prolonged exposures of one state or the other.

Using (3.13), we obtain for this special fitness model when $x_{i}=\omega_{i} z^{i}$ and $y_{j}=\xi_{j} x^{j}$ :

$$
\begin{aligned}
& K=\frac{z\left\{1-(1-\beta) z e^{-\lambda_{1}}-(1-\beta)^{d_{1}}\left(1-e^{-\lambda_{1}}\right) z^{d_{1}}\right\}}{\{1-(1-\beta) z\}\left\{1-(1-\beta) e^{-\lambda_{1} z}\right\}}, \\
& \Lambda=\frac{z\left\{1-(1-\alpha) z e^{-\lambda_{0}}-(1-\alpha)^{d_{0}}\left(1-e^{-\lambda_{0}}\right) z^{d_{0}}\right\}}{\{1-(1-\alpha) z\}\left\{1-(1-\alpha) e^{-\lambda_{0} z}\right\}},
\end{aligned}
$$

which allows us to evaluate the mean individual fitness for large $L$ using (3.6). The mean $\log$ of individual fitness for large $L$ and $\alpha$ and $\beta$ not too close to 0 is

$$
E(\ln W) \simeq \ln C-L\left\{p_{0}(1-\alpha)^{d_{0}} \lambda_{0}+p_{1}(1-\beta)^{d_{1}} \lambda_{1}\right\}
$$

\section{The Genetic Evolution of a Population}

We will now specify our basic genetic constraints. We will limit ourselves to evolution with respect to a single locus with two alleles ( $A$ and $a$ ) in an infinite-sized, random-mating population with discrete generations. In each generation all organisms are subjected to a period of $L$ time units of the environmental process governed by (2.1). Each generation samples an independent realization of this process. We will initially study the situation in which all individuals of a single generation experience the same realization of the environmental process, but later we will examine the case in which all individuals sample independently.

In the case of each generation sampling only a single environmental realization, individual fitness equals genotype fitness and thus the allele frequency is influenced by fitnesses that must be regarded as random variables. We cannot 
properly speak of a selective equilibrium as in a constant fitness model, but we can investigate the fitness conditions that protect an allele from loss. These fitness conditions have already been worked out (e.g., Karlin and Lieberman, 1974; Gillespie, 1973), so our task is to relate them to environmental and organism response patterns.

Let each genotype have a fitness of the form given by (3.2):

$$
\begin{array}{ccc}
A A & A a & a a \\
W_{1} & W_{2} & W_{3} .
\end{array}
$$

In the general nondominant case, the condition for protecting the $A$ allele from loss is (Karlin and Lieberman, 1974; Gillespie, 1973)

$$
E\left(\ln W_{3}\right)<E\left(\ln W_{2}\right) .
$$

Similarly, the condition for protection of the $a$ allele is

$$
E\left(\ln W_{1}\right)<E\left(\ln W_{2}\right) .
$$

We can therefore predict which alleles will be protected by coupling (4.1) and (4.2) to (3.11). In the special case of large $L$ and $\alpha$ and $\beta$ not too close to zero, the conditions for a polymorphism are, using (3.12),

$$
\frac{L \alpha \beta}{\alpha+\beta}\left(\overline{\ln \omega_{2}}+\overline{\ln \xi_{2}}-\overline{\ln \omega_{j}}-\overline{\ln \xi_{j}}\right)<\ln C_{2}-\ln C_{j}, \quad j=1,3 .
$$

The right-hand side of (4.3) represents the fitness differences between heterozygote and homozygote with respect to those factors unrelated to the environmental variation modeled by (2.1). The left-hand side represents the impact of the environmental heterogeneity and the relative homeostatic abilities of the genotypes in coping with this variability. We can now investigate how (4.3) (and thus the evolution of the population) depends upon both the environment and the individual's responses.

From (4.3), we can see that the homeostatic term increases in importance as $L$ (the amount of time influenced by this environment) increases, particularly with respect to the time of the average environmental cycle length $(1 / \alpha+1 / \beta)$. Another reason why the homeostatic term becomes more and more important with increasing $L$ is simply that the probability of experiencing some long runs of 1's or 0's increases with $L$ (although, of course, the probabilities of the runs themselves are independent of $L$ ). This means that the types of fitness effects we are modeling become increasingly important in long-lived (relative to the environmental cycle length) organisms. We would therefore expect selection in long-lived organisms to favor more and more those genotypes with the greatest homeostatic capabilities even if they have lower inherent fitness (the $\ln C$ 's) 
and even if they have lower fitness under the most commonly occurring environmental states (since the rare, long runs now have an increased impact).

From (3.12), we can see that the fitness responses to the environmental runs that enter into the left-hand side of 4.3 are weighted by $(1-\beta)^{n-1}$ for runs of length $n$ of 1 's and $(1-\alpha)^{n-1}$ for 0 's. For $\alpha, \beta \neq 0$, these weights decrease geometrically with run length. Consequently, we would predict that if $(1-\alpha)$ and/or $(1-\beta)$ are small, those genotypes which put their energy into shortterm homeostatic solutions that buffer the organism against short runs would be favored over those genotypes that put their energy into buffering against long runs at the expense of short-term strategies. Since $(1-\alpha)$ and $(1-\beta)$ measure the runniness of the environment (the probability of the previous environmental state persisting), (4.3) implies that selection favors those genotypes that have the best short-term homeostatic capabilities in nonrunny environments. However, as $1-\alpha$ and/or $1-\beta$ increase, the fitness impact of the longer runs increases in importance. Moreover, as seen from (3.11), even $\xi_{L}$ and $\omega_{L}$ (the fitness impact of living all of $L$ under only one environmental state) may become important if $\alpha$ and/or $\beta$ are small enough. Hence, runny environments would favor genotypes that have long-term homeostatic solutions.

More insight into the above statements may be gained by considering the special fitness case described by (3.13). With this model, (4.3) becomes

$$
\begin{aligned}
& L\left(p_{0}\left\{(1-\alpha)^{d_{02}} \lambda_{02}-(1-\alpha)^{d_{0 j}} \lambda_{0 j}\right\}+p_{1}\left\{(1-\beta)^{d_{12}} \lambda_{12}-(1-\beta)^{d_{1 j}} \lambda_{1 j}\right\}\right) \\
& \quad<\ln C_{2}-\ln C_{j}, \quad j=1,3 .
\end{aligned}
$$

Each genotype contributes five parameters to (4.2): $\ln C_{j}$, two $d_{i j}$ 's (short-term response parameters), and two $\lambda_{i j}$ 's (long-term response parameters). We can now investigate the evolutionary impact of the homeostatic parameters as a function of the environmental parameters.

To study the importance of the longterm parameters $\lambda$, let us examine how expected $\log$ fitness (which determines the evolutionary fate of the alleles) depends upon, say, $\lambda_{11}$. From (3.15), we see that the expected log fitness of the $A A$ genotype varies linearly with $\lambda_{11}$ with a slope of

$$
-L p_{1}(1-\beta)^{d_{11}} \text {. }
$$

Equation (4.5) shows that an increase in $\lambda_{11}$ (an increase in the sensitivity to long runs of 1's) depresses the fitness of $A A$ and makes it less likely for $A$ to be fixed. However, just how sensitive the $A A$ 's fitness is to $\lambda_{11}$ depends upon the time spent in the environment $(L)$, the average state frequency of $1\left(p_{1}\right)$, the runniness of the 1's $(1-\beta)$, and the short-term homeostatic ability to withstand 1's $\left(d_{11}\right)$. All of this may be summarized by stating that fitness sensitivity to $\lambda_{11}$ increases with an increase in the average number of l's encountered in the organism's lifetime that diminish fitness; i.e., the average number of 1's 
encountered that were preceded by at least $d_{11}$ previous l's. This is easily seen by returning to the derivation of (3.12) and (3.11), but instead of inserting $\ln \omega_{n}$, substitute an indicator variable that equals zero for all runs of 1 less than or equal to $d_{11}$, and equals 1 for all runs of 1 greater than $d_{11}$. The second term of (3.10) then becomes the expected number of l's encountered during $L$ that diminish fitness. For large $L$ and $\alpha$ and $\beta$ not too close to 0 , this term reduces to (4.5).

As is seen from (4.5), the sensitivity to long runs of 1's is highly dependent upon the short-term homeostatic capabilities since the $d$ 's define the environmental context under which fitness declines. We now will look at the evolutionary impact of the $d$ 's. As (3.15) shows, the expected $\log$ fitness is an exponential function of $d_{1}$; the base of the exponent is $1-\beta$, a measure of the runniness of the environment. This dependence on the runniness is easily seen by plotting (3.15) vs $d_{1}$ for several values of $1-\beta$, as is done in Fig. 1 . If $1-\beta$ is small, a relatively small $d$ will cause a rather large increase in expected log fitness. However, as $1-\beta$ gets larger, the log fitness becomes relatively insensitive to further increases in $\boldsymbol{d}$. Consequently, a favorable fitness strategy in an environment that is not very runny is to increase $d$ slightly; that is, the evolution of homeostatic mechanisms that effectively hide the organism from potentially adverse environments for a short period of time. Of course, this homeostatic strategy is much less effective under runny environments, as Fig. 1 shows. Under these environmental conditions it might be better to achieve homeostasis through a small $\lambda$, since this would represent a long-term solution.

We may now briefly consider the case in which each individual of the popula-

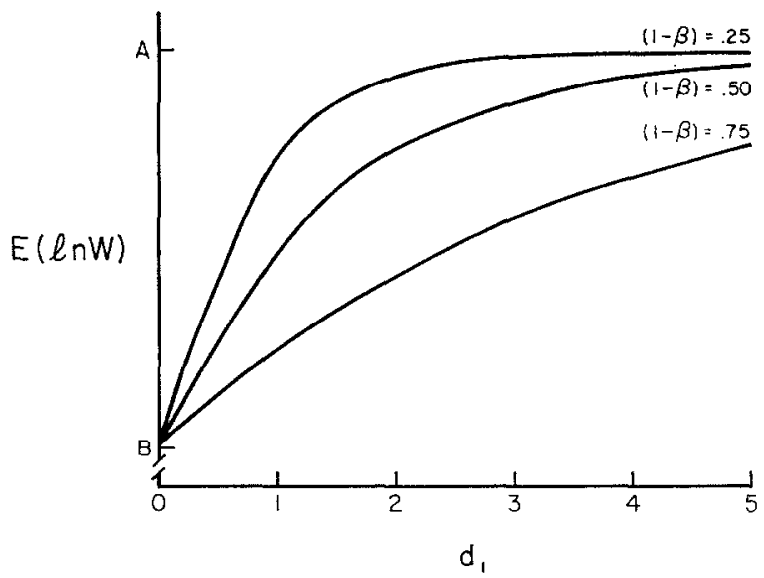

FIG. 1. The expected $\log$ fitness for various values of $1-\beta$ and $d_{1}$ with all other parameters held constant. $L$ is assumed to be large with respect to $(1 / \alpha+1 / \beta)$. The range of $E(\ln W)$ as a function of $d_{1}$ is between $A$ and $B$ where $A=\lim _{d_{1} \rightarrow \infty} E(\ln W)=$ $\ln C-L p_{0} \lambda_{0}(1-\alpha)^{d_{0}}$ and $B=E(\ln W)$ at $d_{1}=0$ which equals $\ln C-L\left[p_{0} \lambda_{0}(1-\alpha)^{d_{0}}+p_{1} \lambda_{1}\right]$. 
tion independently samples the environment described by (2.1). Under these conditions, genotype fitness converges to a constant given by the mean of the individual fitnesses of those sharing a common genotype. Consequently, we may investigate the evolutionary consequences of this fine-grained heterogeneity simply by coupling the expressions for mean fitness given by (3.2) and (3.6) with any of the infinite-sized population genetic models that use constant fitness. In particular, for the one-locus, two-allele random-mating situation previously used, the condition for a polymorphism is now simply mean heterozygote fitness superiority. In general this is a more restrictive condition than that given by (4.1) and (4.2), so we may conclude that correlated sampling by individuals of fine-grained environmental heterogenity makes it more likely to maintain a polymorphism. This may also be interpreted as an increase in the evolutionary chances for greater homeostasis in populations with a history of correlated sampling as contrasted with an equivalent population with independent individual sampling.

For large $L$, the condition for maintaining a polymorphism is, from (3.6\%),

$$
C_{j} \Phi_{j}\left(z_{1 j}\right) z_{1 j}^{-(L+1)}<C_{2} \Phi_{2}\left(z_{12}\right) z_{12}^{-(L+1)}, \quad j=1,3 .
$$

Since $z^{-(L+1)}$ is a decreasing function of $L$, the conclusion we made earlier about the importance of homeostasis increasing with increasing $L$ remains valid. Moreover, all the qualitative statements we made concerning long-term homeostasis, short-term homeostasis, and environmental runniness are also valid for the independent sampling case. This is seen by considering the special case (3.13) and noting that (3.6) is an increasing function of $K$ and $A$. From (3.14), both $K$ and $\Lambda$ are decreasing functions of the appropriate $\lambda$ 's and increasing functions of the appropriate $d$ 's, just as expected log fitness was. Moreover, if $\lambda_{1}$, say, is small, then

$$
K \simeq \frac{z}{1-(1-\beta) z}\left(1-\frac{\lambda_{1}(1-\beta)^{d_{1}} z_{1}^{d_{1}}}{1-(1-\beta) z}\right)
$$

Hence, $K$ (and similarly $\Lambda$ ) is approximately a linear function of $\lambda_{1}$ when $\lambda_{1}$ is small, and once again $d_{1}$ appears as the exponent of the runniness. Consequently, we may extend to the case of independent sampling our prediction that shortterm homeostatic mechanisms tend to evolve in nonrunny environments while long-term mechanisms are favored in runny environments.

\section{Discussion}

If heterozygotes have superior homeostatic abilities, as some evidence indicates (Lerner, 1954), then the type of environmental heterogeneity we are modeling may be an important mechanism in maintaining polymorphisms, 
even if there is no heterozygote superiority under the most commonly occurring environmental conditions. As an example, consider the case in which individuals are sensitive to runs of 1's greater than length 5 , and thereafter fitness falls off at a rate of .04 for $A a$ 's and .05 for $A A$ and $a a$, and all other fitness components are the same for all genotypes. Let the environmental transition matrix be

$$
\left(\begin{array}{ll}
\frac{5}{8} & \frac{3}{8} \\
3 & \frac{1}{4}
\end{array}\right)
$$

and let $L=100$. The expected proportion of an individual's time spent in 1 is $1 / 3$ with a standard deviation of .042 . But because this environment is not very runny, there is only about a $4 \%$ chance that any given sequence of 100 time units will contain any "bad" l's at all (i.e., l's preceded by at least five other l's). In other words, $96 \%$ of the time the environmental conditions are such that heterozygotes and homozygotes appear to have equal fitnesses; yet both (4.4) and (4.6) predict the maintenance of a polymorphism in this case. Consequently, buffering against rarely occurring environmental runs may be an important evolutionary constraint, and one that might often go undetected.

'This same conclusion was reached by Schwartz (1969) as a result of his studies on the alcohol dehydrogenase $\left(\Lambda \mathrm{dh}_{1}\right)$ locus in maizc. We will now consider Schwartz' work in more detail to illustrate how our model could potentially be applied to actual data sets. In maize, alcohol dehydrogenase occurs in the kernel and is active until shortly after germination. Schwartz isolated a mutant at the $\mathrm{Adh}_{1}$ locus that when homozygous fails to produce an active enzyme. This allele is give the symbol $\mathrm{Adh}_{1}{ }^{0}$, and the active allele the symbol $A_{d h}{ }_{1}$. With respect to Adh activity, $A_{d h}$ is a dominant allele. Under aerobic germination conditions, the $\mathrm{Adh}_{1}{ }^{0} / \mathrm{Adh}_{1}{ }^{0}$ homozygous kernels do just as well as $A_{d h} /$-kernels, but Adh is known to be important in energy production in maize under anaerobic conditions. In nature, anaerobic conditions would result from an excessive amount of water in the soil following a heavy rainfall. Under flooding conditions, the recessive Adh-null kernels no longer did well. If subjected to 72 hours of continuous soaking, all of them failed to germinate. $\mathrm{Adh}_{1} /$-kernels, however, were insensitive to this soaking. This type of fitness pattern may be described as

$$
\begin{array}{lccc}
\text { Genotype: } & \operatorname{Adh}_{\mathbf{1}} / \operatorname{Adh}_{1} & \operatorname{Adh}_{1} / \operatorname{Adh}_{1}{ }^{0} & \operatorname{Adh}_{1}{ }^{0} / \operatorname{Adh}_{1}{ }^{0}, \\
\text { Fitness: } & 1 & 1 & W=C \prod_{i} \omega_{n_{i}},
\end{array}
$$

where environmental state 1 now corresponds to the environmental condition of water-saturated soil and $L$ refers to the number of hours (or other convenient time unit) that a kernel spends in the soil before germination. If the population of interest is a large, flat field of maize, then the flooding conditions would most probably be sampled in the identical fashion by all plants. Thus, the random 
fitness model is appropriate, and the fixation of the $\mathrm{Adh}_{\mathbf{1}}{ }^{0}$ allele is stochastically locally stable when $E(\ln W)>0$, and the fixation of the $\mathrm{Adh}_{1}$ allele is stochastically locally stable when $E(W)<1$ (Karlin and Lieberman, 1974). $W$ is of the form given by (3.1), and its parameters are potentially estimable. The work of Schwartz already indicates that $C=1$ and that $\omega_{n}=0$ for $n \geqslant 72$ hours. To estimate the remaining $\omega_{n}$ 's it would be necessary to repeat Schartz' experiments, but with soaking the kernels for a series of time periods less than 72 hours. The estimation of the $\omega_{n}$ 's would be simplified if some simple mathematical function, such as (3.13), could be fitted to the data. Once the $\omega_{n}$ 's are estimated, we could then make predictions about the evolutionary fate of a maize population when subjected to various runs of flooding conditions. Furthermore, other alleles are known at the $\mathrm{Adh}_{1}$ locus that differentially affect survivorship under flooding conditions in maize (Marshall et al., 1973) and perhaps at the homologous locus in other species (Torres et al., 1977). Similar estimation experiments could be done with these other species and alleles, so that this system is not just limited to the dominant case discussed above.

\section{REFERENCES}

Billingsley, P. 1961. "Statistical Inference for Markov Process," Univ. of Chicago Press, Chicago.

FeLler, W. 1957. "An Introduction to Probability Theory and Its Applications," Vol. I, 2nd ed., Wiley, New York.

Felsenstein, J. 1976. The theoretical population genetics of variable selection and migration, Annu. Rev. Genet. 10, 253-280.

Gabriel, K. R. AND NeUmanN, J. 1962. A Markov chain model for daily rainfall occurrence at Tel Aviv, Quart. J. Roy. Meteorol. Soc. 88, 90-95.

Gillespie, J. 1973. Polymorphism in random environments, Theor. Pop. Biol. 4, 193-195.

Goon, I. J. 1973. The joint probability generating function for run-lengths in regenerative binary Markov chains, with application, Ann. Statist. 1, 973-939.

Hedrick, P. W., Ginevan, M. E., and Ewing, E. P. 1976. Genetic polymorphism in heterogeneous environments, Ann. Rev. Ecol. Syst. 7, 1-32.

KARLIN, S. AND LIEBERMAN, U. 1974. Random temporal variation in selection intensities: Case of large population size, Theor. Pop. Biol. 6, 355-382.

LARCHER, W. 1975. "Physiological Plant Ecology," Springer-Verlag, Berlin.

Lerner, I. M. 1954. "Genetic Homeostasis," Dover, New York.

Mangum, C. AND Towle, D. 1977. Physiological adaptation to unstable environments, Amer. Sci. 65, 67-75.

Marshall, D. R., Brove, P., And Pryor, A. J. 1973. Adaptive significance of alcohol dehydrogenase isozymes in maize, Nature New Biol. 244, 16-18.

Martin, J. J. 1967. "Bayesian Decision Problems and Markov Chains," Wiley, New York.

Schwartz, D. 1969. An example of gene fixation resulting from selective advantage in suboptimal conditions, Amer. Natur. 103, 479-481.

Strobeck, C. 1975. Selection in a fine-grained environment, Amer. Natur. 109, 419-425.

Templeton, A. R. 1977. Survival probabilities of mutant alleles in fine grained environments, Amer. Natur. 111, 951-966. 
Templeton, A. R. and Rothman, E. D. 1978. Evolution and fine-grained environmental runs, in "Foundations and Applications of Decision Theory" (C. A. Hooker, Ed.), Reidel, Dordrecht, in press.

Torres, A. M., Diedentofen, U., and Johnstone, I. M. 1977. The early allele of alcohol dehydrogenase in sunflower populations, J. Hered. 68, 11-16. 